\title{
A novel TFT-OLED integration for OLED-independent pixel programming in amorphous-Si AMOLED pixels
}

\author{
Bahman Hekmatshoar \\ Alex Z. Kattamis \\ Kunigunde Cherenack \\ Sigurd Wagner \\ James C. Sturm
}

\begin{abstract}
The direct voltage programming of active-matrix organic light-emitting-diode (AMOLED) pixels with $n$-channel amorphous-Si (a-Si) TFTs requires a contact between the driving TFT and the OLED cathode. Current processing constraints only permit connecting the driving TFT to the OLED anode. Here, a new "inverted" integration technique which makes the direct programming possible by connecting the driver n-channel a-Si TFT to the OLED cathode is demonstrated. As a result, the pixel drive current increases by an order of magnitude for the same data voltages and the pixel data voltage for turn-on drops by several volts. In addition, the pixel drive current becomes independent of the OLED characteristics so that OLED aging does not affect the pixel current. Furthermore, the new integration technique is modified to allow substrate rotation during OLED evaporation to improve the pixel yield and uniformity. The new integration technique is important for realizing active-matrix OLED displays with a-Si technology and conventional bottom-anode OLEDs.
\end{abstract}

Keywords - a-Si, active matrix, AMOLED display, TFT-OLED integration, bottom-anode OLEDs.

\section{Introduction}

Superior properties of organic light-emitting diodes (OLEDs) such as high-speed response, emissivity, wide viewing angle, simple structure, and anticipated low fabrication cost make them very appealing for display applications. ${ }^{1}$ Integrating OLEDs with TFTs in the form of active matrices is required for achieving low power consumption in mid- and largesized displays. ${ }^{2,3}$ Although amorphous-Si (a-Si) technology is low in cost, in widespread production, and very suitable for large-area deposition especially on flexible substrates, low-temperature poly-Si (LTPS) has been the first material of choice for TFT backplanes since the introduction of AMOLED displays. The advantages of LTPS over a-Si are (i) higher TFT mobility, (ii) higher TFT stability, and (iii) availability of $p$-channel TFTs. ${ }^{2,4}$ Although using a-Si for
AMOLED applications has been demonstrated ${ }^{5,6}$ and complete a-Si AMOLED displays have been realized by industry, ${ }^{7,8}$ the commercial production of AMOLED displays requires that weaknesses of a-Si be resolved or effectively compensated. The low field-effect mobility in a-Si may be compensated for by developing high-efficiency OLED's which require low driving currents. ${ }^{9}$ The instability of threshold voltage in a-Si TFTs is especially serious when they are fabricated at low process temperatures compatible with the typical flexible clear plastic substrates. The reliability of a-Si TFTs can be improved by using clear plastic substrates which allow higher process temperatures ${ }^{10}$ or by circuits which compensate for threshold voltage shift. ${ }^{11}$ Using more-efficient OLEDs also alleviates the a-Si TFT stability problem, because the threshold voltage shift is lower at lower driving currents and lower gate voltages. ${ }^{9} \mathrm{~A}$

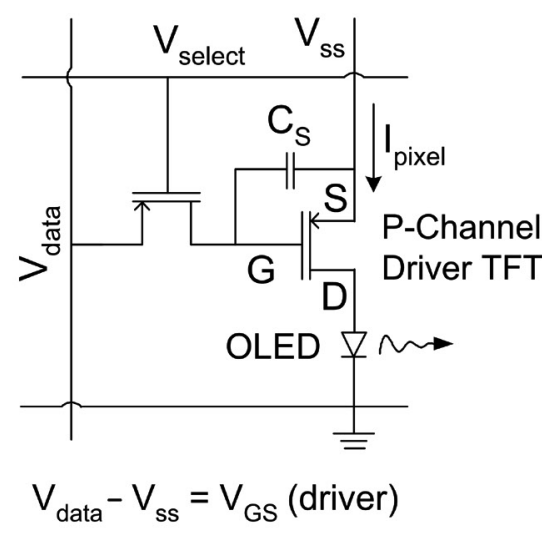

(a)

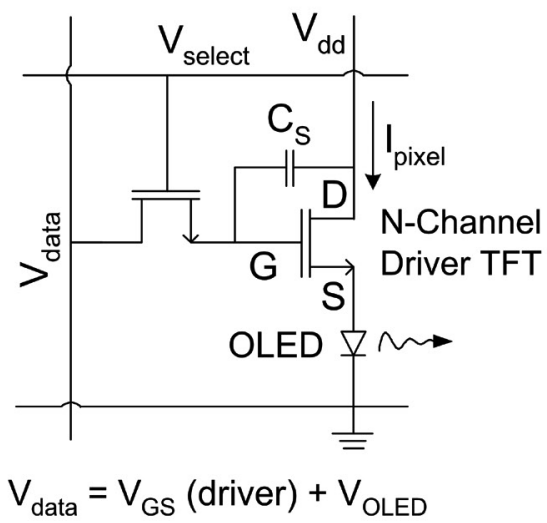

(b)

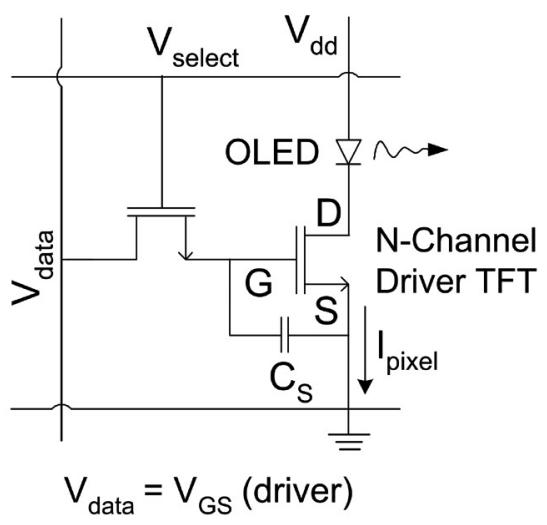

(c)

FIGURE 1 - Circuit schematic of 2-TFT AMOLED pixels: (a) conventional structure with p-channel TFTs (low-temperature poly-Si), (b) conventional structure with $n$-channel TFTs (a-Si), and (c) new "inverted" structure with $n$-channel TFTs (a-Si).

The authors are with Princeton University, Princeton Institute for the Science and Technology of Materials (PRISM) and the Department of Electrical Engineering, Olden St., Princeton, N.J. 08540; telephone 609/258-6624, fax -1840, e-mail: hekmat@princeton.edu.

(c) Copyright 2008 Society for Information Display 1071-0922/08/1601-0183\$1.00 
serious issue with a-Si TFT pixel circuits is the direct programming of the pixel current by the data voltage, which is not conventionally possible in a-Si technology due to the lack of $p$-channel TFTs. ${ }^{2,4}$ Addressing this issue is the focus of this paper.

Figure 1(a) shows the circuit schematic of a conventional 2-TFT AMOLED pixel fabricated in LTPS technology using conventional TFT-OLED integration with $p$-channel poly-Si TFTs. The pixel cross section is shown in Fig. 2. The conventional integration sequence is dictated by three constraints: $(i)$ the OLEDs must be evaporated after the TFT fabrication process because the TFT process severely damages the OLEDs; (ii) the best OLEDs are deposited from anode to cathode, i.e., the anode (e.g., ITO) is deposited first, followed by the organic layers and then the cathode (bottom-anode OLEDs); and (iii) patterning the organic layers and cathode by photolithography is generally not feasible without damaging the OLEDs. As a result, the driver TFT is connected to the OLED anode rather that the

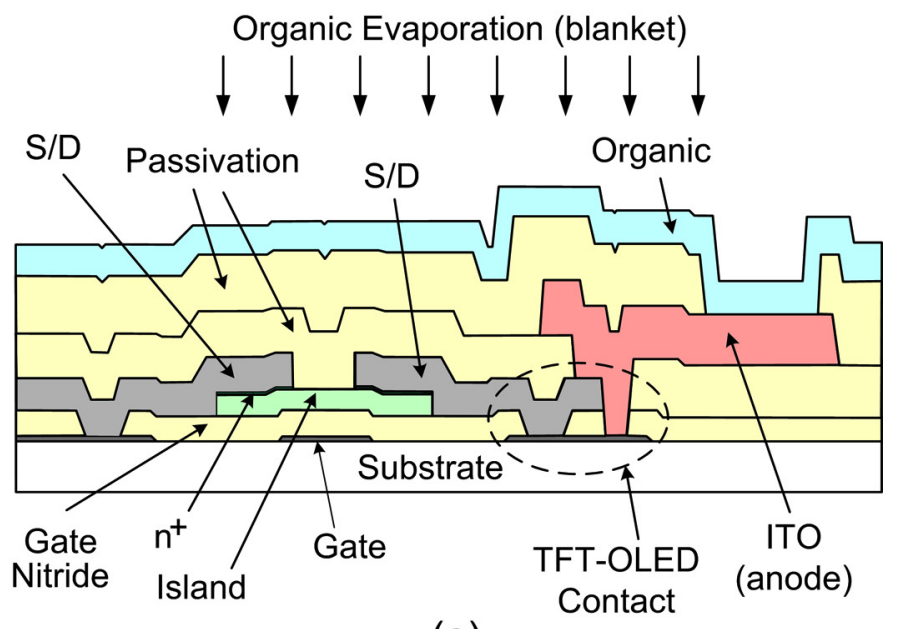

(a)

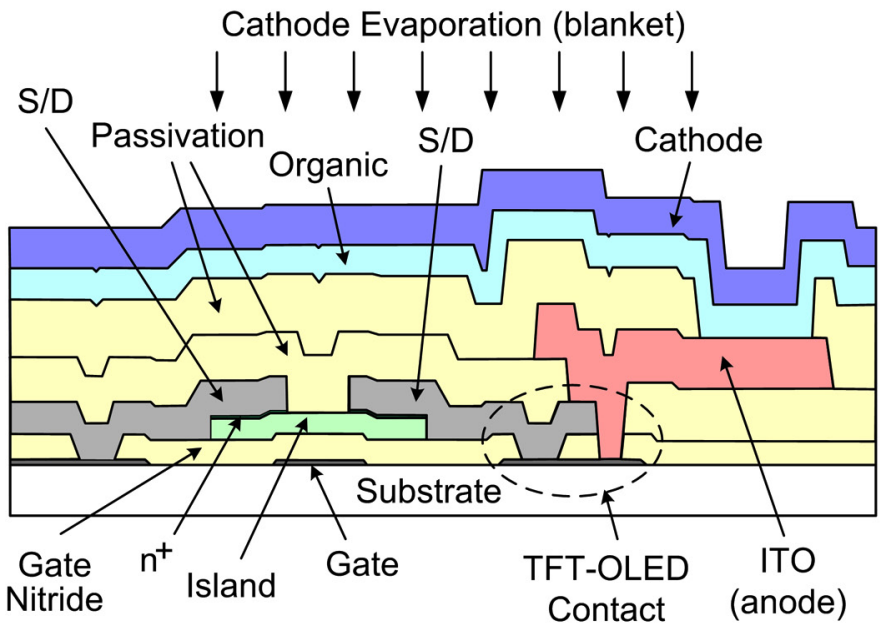

(b)

FIGURE 2 - Schematic cross section of the fabricated conventional AMOLED structure [used in the pixel circuits of Figs. 1(a) and 1(b)] during the evaporation of (a) the organic layers and (b) cathode.
OLED cathode (Fig. 2). With $p$-channel TFTs [Fig. 1(a), LTPS AMOLED pixels], the TFT terminal connected to the OLED is the drain, and therefore the gate-source voltage of the driver TFT is determined directly by the data voltage $\left(V_{\text {data }}\right)$ and is independent of the OLED characteristics. This is because the TFT current in saturation is controlled by $V_{\text {Gate }}-V_{\text {Source, }}$ or in this case $V_{\text {data }}-V_{S S}\left(V_{S S}\right.$ is a fixed voltage).

If the conventional integration (Fig. 2) is used for a-Si technology where only $n$-channel TFTs are available, the TFT terminal connected to the OLED will be the TFT source [Fig. 1(b), conventional a-Si AMOLED pixels]. Therefore the data voltage is split across the OLED and the gate source of the driving TFT $\left[V_{\text {data }}=V_{G S}(\right.$ driver $\left.)+V_{\text {OLED }}\right]$. This is not desirable for two reasons: $(i)$ higher data voltages are required for programming the pixel to obtain the same pixel currents [requiring the same $V_{\mathrm{GS}}$ (driver)], because a part of the data voltage drops across the OLED rather than dropping entirely across the gate source of the driving TFT; and $(i i)$ the voltage drop across the gate source of the driving TFT and thus the pixel current depends on the OLED characteristics, which may vary device to device in manufacturing and vary with time during device operation. Therefore, direct programming of a-Si TFTs requires a new technique for connecting the driver TFT to the OLED cathode instead of the OLED anode, as shown in Fig. 1(c), so that the data voltage may be transferred directly to the gate source of the driver TFT. Such an integration technique is presented in this work.

\section{Fabrication}

The schematic cross section of an a-Si AMOLED pixel fabricated with the inverted integration process is shown in Fig. 3. The cross section corresponds to the circuit sche-
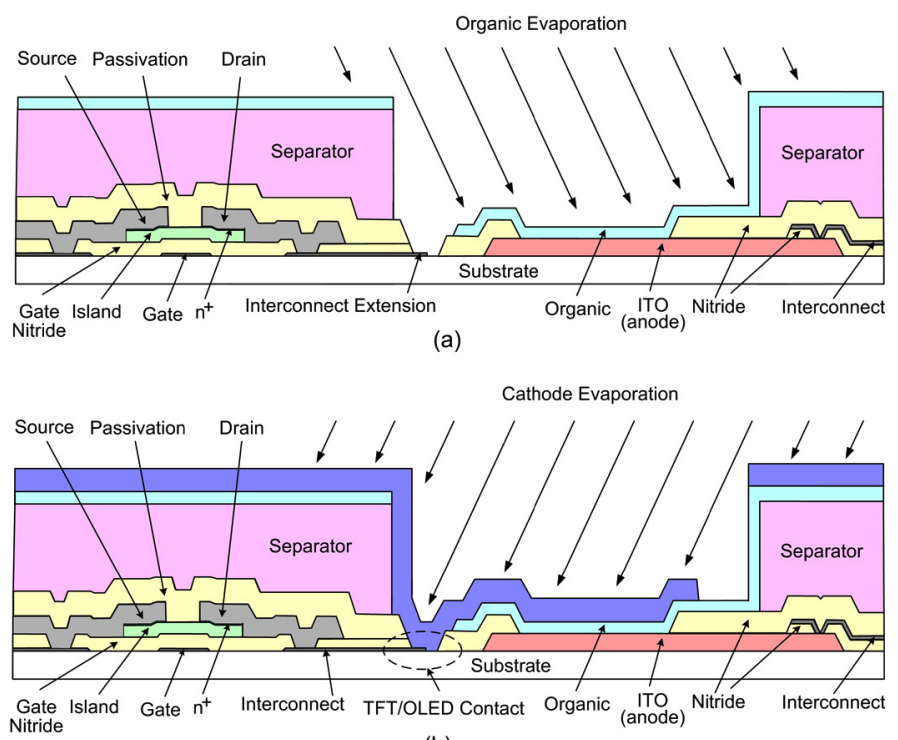

(b)

FIGURE 3 - Schematic cross section of the fabricated new "inverted" AMOLED structure of Fig. 1(c), during the evaporation of (a) the organic layers and (b) cathode. 
matic of Fig. 1(c). The a-Si TFT backplane is fabricated at temperatures up to $300^{\circ} \mathrm{C}$ on glass. ${ }^{5}$ The apparent (i.e., not corrected for contact resistance) saturation mobility and threshold voltage of the driving TFTs $(L=5 \mu \mathrm{m})$ are $0.65 \pm$ $0.04 \mathrm{~cm}^{2} / \mathrm{V}$-sec and $1.7 \pm 0.2 \mathrm{~V}$, respectively. After processing the TFT backplane (including ITO as the OLED anode), insulating "separators" are formed by patterning a layer of positive photoresist using conventional photolithography. As shown in Fig. 3(a), the organic layers are then evaporated at an angle in such a way that an interconnect extension connected to the driving TFT is not coated with the organic layers, taking advantage of the separator's shadowing effect. We have used $10-\mu \mathrm{m}$-thick photoresist separators and stand-

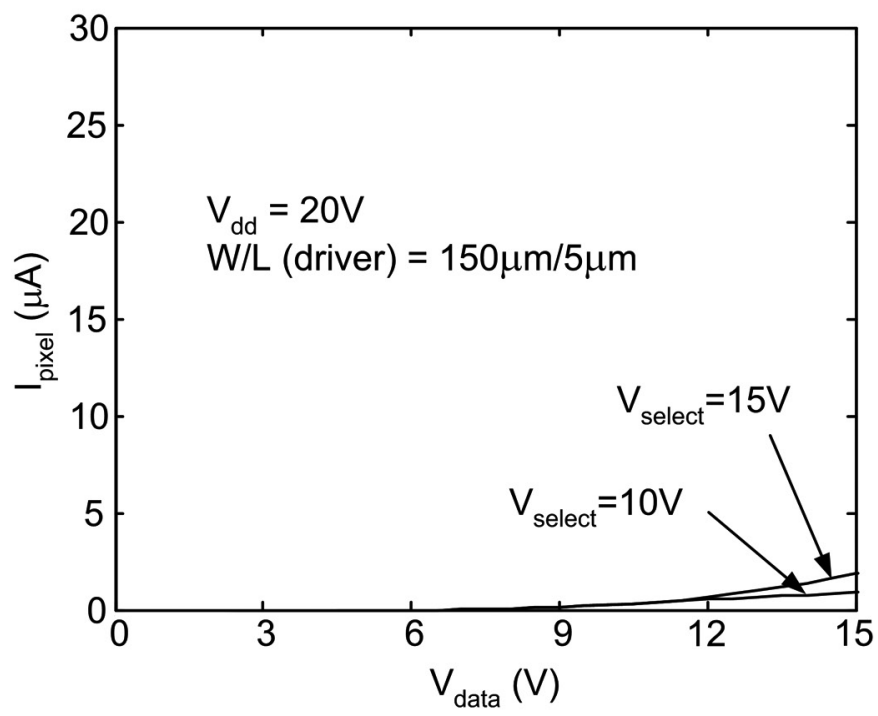

(a)

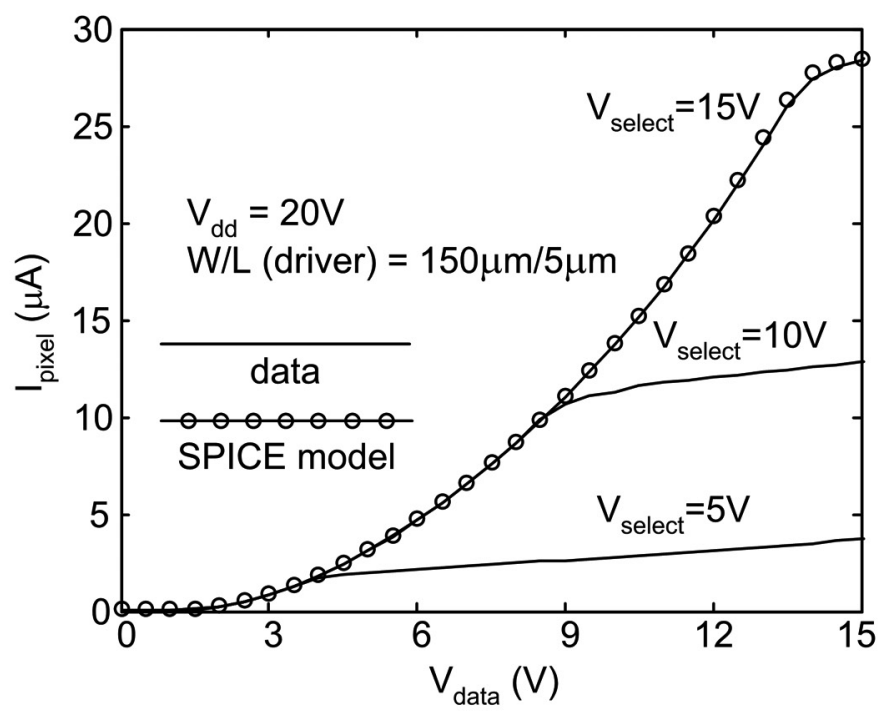

(b)

FIGURE 4 - Measured pixel current $\left(I_{\text {pixel }}\right)$ as a function of the programmed data voltage $\left(V_{\text {data }}\right)$ of (a) a conventional a-Si AMOLED pixel shown in the pixel circuit of Fig. $1(\mathrm{~b})$ and $1(\mathrm{~b})$ an inverted a-Si AMOLED pixel shown in the pixel circuit of Fig. 1(c). The SPICE simulation for $V_{\text {select }}=15 \mathrm{~V}$ is also plotted in (b). ard TPD/ALq3 organic layers for this experiment. Then, as shown in Fig. 3(b), the cathode $(\mathrm{Mg}-\mathrm{Ag} / \mathrm{Ag})$ is evaporated at an angle opposite to the organic evaporation angle to form the OLED cathode and also to contact the interconnect extension. Therefore, the electrical circuit of Fig. 1(c) is realized.

\section{TFT-OLED integration results}

The measured dc characteristics of a-Si AMOLED pixels integrated with the conventional and inverted processes are compared in Fig. 4. First, in the inverted structure [Fig. $4(\mathrm{~b})$ ], the pixel drive current, $I_{\text {pixel }}$, turns on at $V_{\text {data }}=1.7 \mathrm{~V}$ (corresponding to the threshold voltage of the driver TFT) which is considerably lower than the conventional design

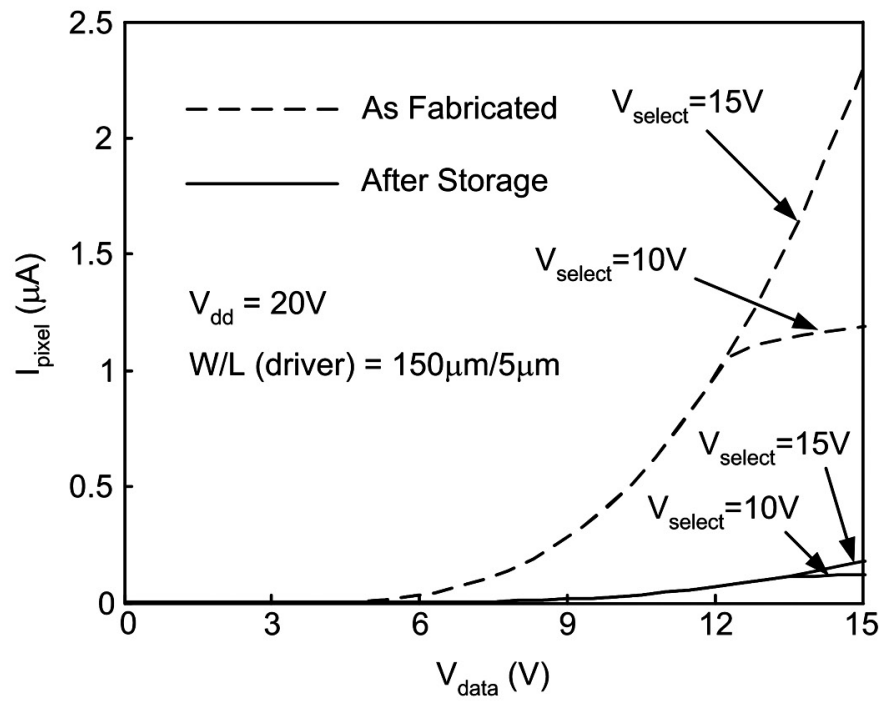

(a)

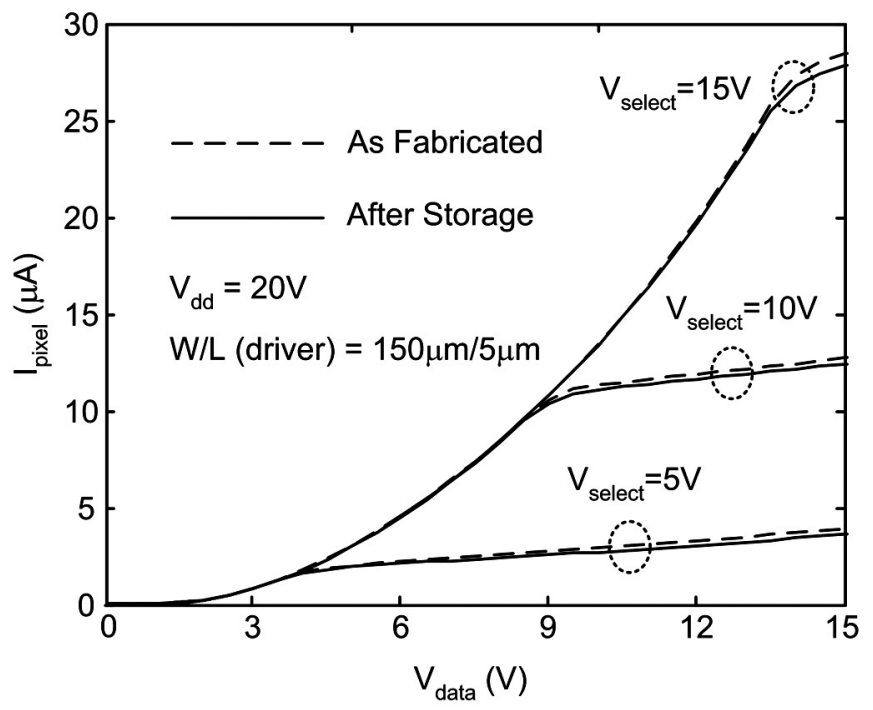

(b)

FIGURE 5 - The effect of the drift in OLED characteristics caused by storing unencapsulated devices in an environment relatively high in oxygen and humidity, on the pixel driving current for (a) conventional and (b) inverted AMOLED pixels. 


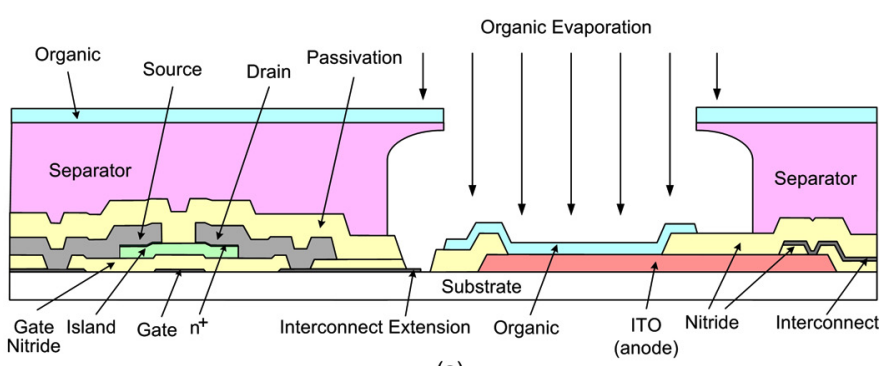

(a)

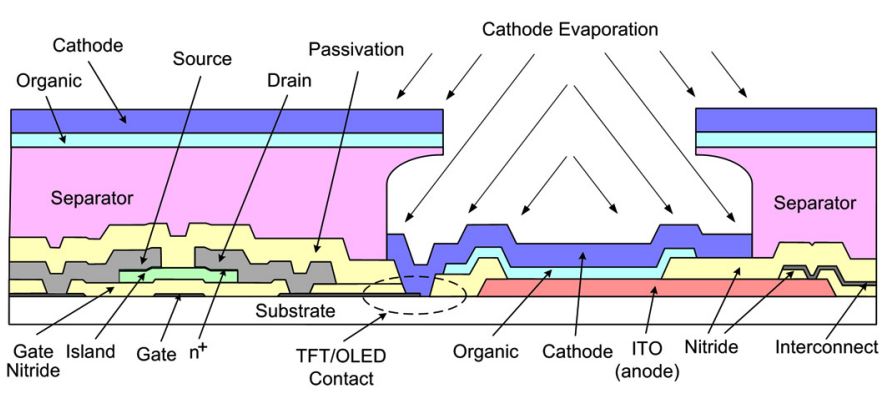

(b)

FIGURE 6 - Schematic cross section of the "modified" inverted AMOLED pixel, during the evaporation of (a) the organic layers and (b) cathode.

[Fig. 4(a)], where $I_{\text {pixel }}$ turns on at $V_{\text {data }}=4.8 \mathrm{~V}$ [corresponding to the threshold voltage of the driving TFT $(1.7 \mathrm{~V})$ plus the turn-on voltage of the OLED (3.1 V)]. Second, in the inverted structure, at typical operational current levels of a few microamperes, the pixel current is higher by an order of magnitude than the current in the conventional structure for the same data voltages. This is because in the conventional design the data voltage is split across the OLED and the gate source of the driving TFT, but in the inverted design it is converted directly to the gate-source voltage of the driving TFT. SPICE simulations confirm the experimental behavior of the inverted pixels [Fig. 4(b)].

To further verify the independence of the pixel driving current from the OLED characteristics, we compared the drift in the output characteristics of conventional and inverted AMOLED pixels after storing them in a non-ideal environment. The AMOLED arrays, which were not encapsulated, were stored in a nitrogen box with a relatively high oxygen content of about $100 \mathrm{ppm}$, for 6 months. The storage condition will not alter a-Si TFTs, but the oxygen content and humidity lead to considerable OLED degradation. Figure 5 (a) shows a large drop in $I_{\text {pixel }}$ of conventional AMOLED pixels after storage. This is because the OLED degradation causes an increase in the voltage drop across the OLED for a given current, and thus a higher voltage is required to achieve the same $V_{\mathrm{GS}}\left(\right.$ driver) and the same $I_{\text {pixel }}$ in the driver TFT. In contrast, $I_{\text {pixel }}$ of inverted AMOLED pixels [Fig. 5(b)] is not affected by OLED degradation, an observation verifying that $I_{\text {pixel }}$ is independent of the OLED characteristics (provided that $V_{\mathrm{dd}}$ is high enough to ensure the driver TFT is still in saturation).
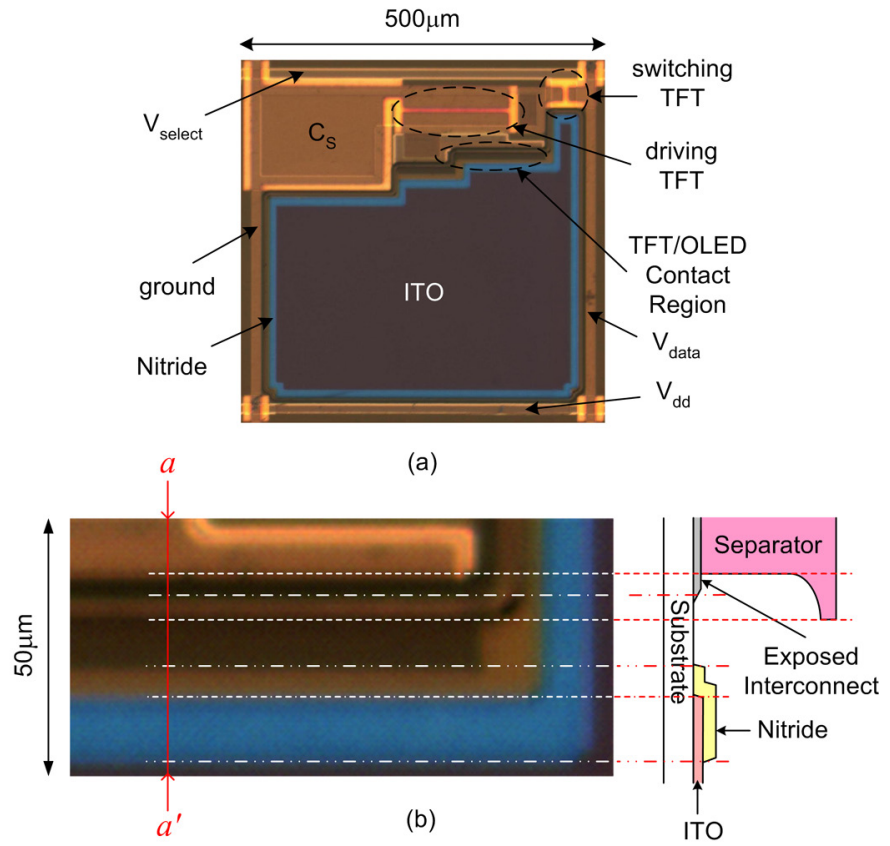

FIGURE 7 - (a) Optical micrograph of a modified inverted pixel (Fig. 6) prior to OLED evaporation and (b) higher magnification of the TFT-OLED contact region along with schematic cross section along line $a-a^{\prime}$. The non-modified inverted structure (Fig. 3 ) has the same geometry, except for the separator which lacks the overhang.

\section{Modified integration}

Although the integration process introduced in Fig. 3 realizes the inverted structure of Fig. 1(c) and makes direct programming of the pixel current possible, it is prone to pixel yield loss and non-uniformity because it does not allow for substrate rotation during the evaporation of organic layers and the cathode. To overcome this problem, we have modified the integration process by using insulating separators with an overhanging projection (Fig. 6) using a double-layer photoresist process. Implementation with other methods may be possible as well. In our experiment, we have used $10-\mu \mathrm{m}$-high separators with $5 \mu \mathrm{m}$ of overhang. As shown in Fig. 6(a), the organic layers are then evaporated at normal incidence and the substrate is rotated during organic evaporation. The overhang shadows an exposed interconnect

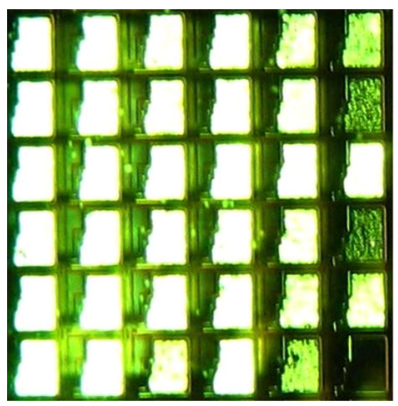

(a)

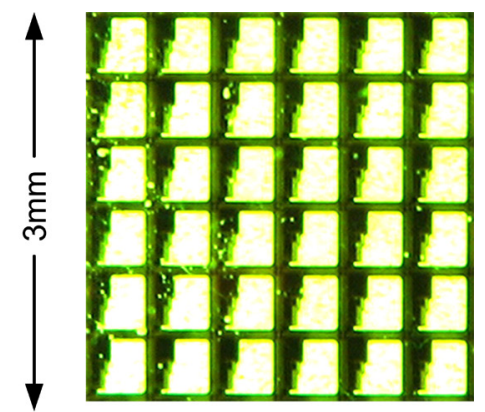

(b)
FIGURE 8 - Comparison of the AMOLED pixels fabricated by the inverted process of Fig. 3 (no rotation) and the modified inverted process of Fig. 6 (with rotation). 


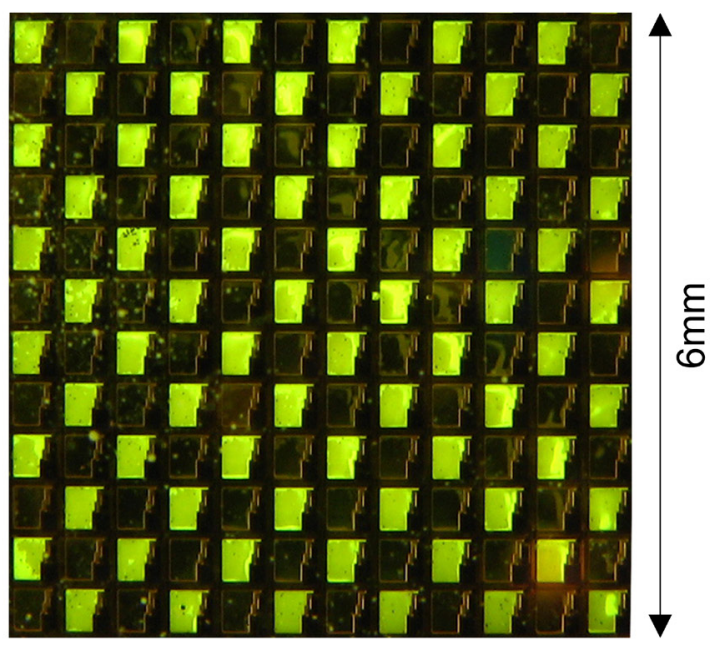

(a)

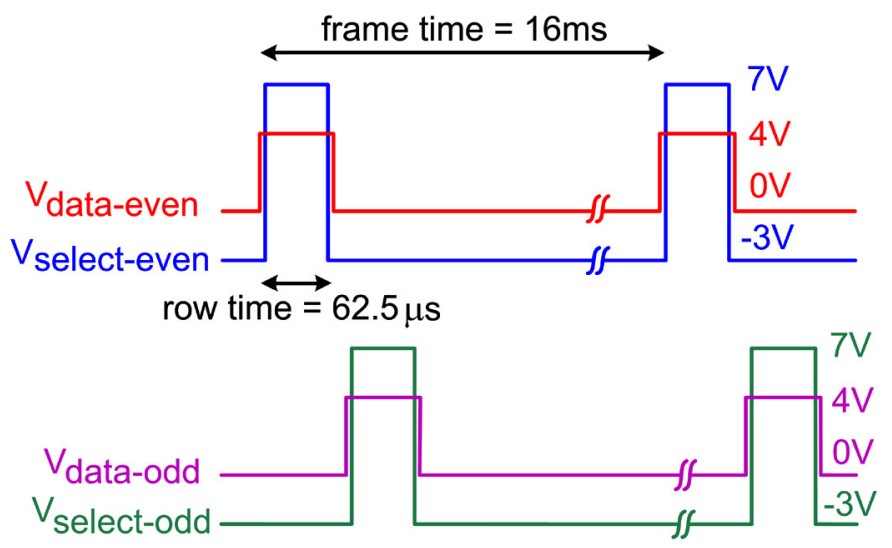

(b)

FIGURE 9 - (a) QVGA checkerboard demonstration of the AMOLED pixels fabricated by the modified inverted process presented in Figs. 6(a) and $6(b)$ the drive signals.

which is connected to the driver TFT. The cathode is evaporated next at an angle while the substrate is being rotated [Fig. 6(b)], and therefore the OLED cathode is connected to the exposed interconnect and the inverted structure of Fig. 1(c) is realized. The cathode may be also evaporated at multiple angles for maximum step coverage.

Figure 7 shows an optical micrograph of a modified inverted pixel prior to organic and cathode evaporation. The ITO area, excluding its passivated edges, defines the pattern of emission. AMOLED test arrays fabricated using the inverted integration process shown in Fig. 3 and the modified inverted process shown in Fig. 6 are compared in Figs. $8(\mathrm{a})$ and $8(\mathrm{~b})$, respectively. It is observed that the modified inverted process results in a higher pixel yield and better uniformity. A quarter video graphics array (QVGA) checkerboard demonstration of a $12 \times 12$ AMOLED test array fabricated using the modified inverted integration is presented in Figs. 9(a) and 9(b) as a proof of high pixel yield and uniformity.

\section{$5 \quad$ Summary and conclusion}

In summary, we have demonstrated the direct programming of a-Si TFT AMOLED pixels using a new integration technique that connects the OLED top contact (cathode) to the underlying TFT. We have shown that by using a new "inverted" integration process the drive current of the fabricated pixels becomes essentially independent of the OLED characteristics and therefore is not affected by OLED aging. Furthermore, as a result of direct programming, the data voltages required for typical pixel operation currents (on the order of $1 \mathrm{~mA} / \mathrm{cm}^{2}$ ) drop from about $15 \mathrm{~V}$ to about $5 \mathrm{~V}$. The pixel yield is increased and the uniformity is improved by introducing a modified version of the inverted integration process which allows substrate rotation during OLED evaporation. This integration approach to the direct programming of a-Si AMOLED pixels may be important for the realization of AMOLED displays with a-Si TFT backplanes.

\section{Acknowledgment}

The authors would like to thank the Dupont Company for technical collaboration. This work is sponsored by the U.S. Display Consortium through the project on $300^{\circ} \mathrm{C}$ Amorphous TFT Display Backplane Processes on Clear Plastic Substrates.

\section{References}

1 R. Dawson, Z. Shen, D. A. Furst, S. Connor, J. Hsu, M. G. Kane, R.G Stewart, A. Ipri, C. N. King, P. J. Green, R. T. Flegal, S. Pearson, W. A. Tang, S. Van Slyke, F. Chen, J. Shi, M. H. Lu, and J. C. Sturm, "The impact of the transient response of organic light emitting diodes on the design of active matrix OLED displays," Tech. Dig. IEEE Electron Dev. Meeting, 875-878 (1998).

2 M. Hack, J. J. Brown, J. K. Mahon, R. C. Kwong, and R. Hewitt, "Performance of high-efficiency AMOLED displays," J. Soc. Info. Display 9, No. 3, 191-195 (2001)

3 R. Dawson, M. G. Kane, Z. Shen, D. A. Furst, S. Connor, J. Hsu, R. G. Stewart, A. Ipri, C. N. King, P. J. Green, R. T. Flegal, S. Pearson, W. A. Barrow, E. Dickey, K. Ping, S. Robinson, C. W. Tang, S. Van Slyke, F. Chen, J. Shi, J. C. Sturm, and M. H. Lu, "Active matrix organic light emitting diode pixel design using polysilicon thin film transistors," Conf. Proc. Laser Electron Opt. Soc. Annual Meet. LEOS 1, 128-129 (1998)

4 J. Lih, C. Sung, C. Li, T. Hsiao, and H. Lee, "Comparison of a-Si and poly-Si for AMOLED displays," J. Soc. Info. Display 12, No. 4 , 367-371 (2004).

5 C. C. Wu, S. D. Theiss, G. Gu, M. H. Lu, J. C. Sturm, S. Wagner, and S. R. Forrest, "Integration of organic LEDs and amorphous Si TFTs onto flexible and lightweight metal foil substrates," IEEE Electron Dev. Lett. 18, No. 12, 609-612 (1997).

6 M. H. Lu, E. Ma, J. C. Sturm, and S. Wagner, "Amorphous silicon TFT active-matrix OLED pixel," Conf. Proc. Laser Electron Opt. Soc. Annual Meet. LEOS 1, 130-131 (1998).

7 T. Tsujimura, Y. Kobayashi, K. Murayama, A. Tanaka, M. Morooka, E Fukumoto, H. Fujimoto, J. Sekine, K. Kanoh, K. Takeda, K. Miwa, M Asano, N. Ikeda, S. Kohara, S. Ono, C. Chung, R. Chen, J. Chung, C.-W. Huang, H. Guo, C. Yang, C. Hsu, H. Huang,W. Riess, H. Riel, S. Karg, T. Beierlein, D. Gundlach, S. Alvarado, C. Rost, P. Muller, F Libsch, M. Mastro, R. Polastre, A. Lien, J. Stanford, and R. Kaufman, "A 20-inch OLED displays driven by super-amorphous silicon technology," SID Symposium Digest Tech. Papers 34, 6-9 (2003).

8 J. Lih and C. Sung, "Full-color active-matrix OLED based on a-Si TFT technology," J. Soc. Info. Display 12, No. 4, 367-371 (2004). 
9 J.-J. Lih, C-F. Sung, M. S. Weaver, M. Hack. and J. J. Brown, "A phosphorescent active-matrix OLED display driven by amorphous silicon backplane," J. Soc. Info. Display 11, No. 1, 14-17 (2003).

10 K. Long, A. Kattamis, I.-C. Cheng, H. Gleskova, S. Wagner, and J. C. Sturm, "Stability of amorphous-silicon TFTs deposited on clear plastic substrates at $250^{\circ} \mathrm{C}$ to $280^{\circ} \mathrm{C}$," IEEE Electron Dev. Lett. 27, No. 2, 111-113 (2006).

11 A. Nathan, A. Kumar, K. Sakariya, P. Servati, K. S. Karim, D. Striakhilev, and A. Sazonov, "Amorphous silicon back-plane electronics for OLED displays," IEEE J. Sel. Top. Quantum Electron. 10, No. 1, 58-69 (2004). 